Bowel Cancer Screening Programme offers colonoscopy to subjects aged 60-74 after a positive faecal occult blood test (FOBT). All colonoscopists have to be screening-accredited with a lifetime log of over 1000 colonoscopies with certain minimum key performance indicators and then undergo a formal structured assessment process during which they are observed performing 2 colonoscopies by trained assessors.

Methods The aim was to assess how performance varied in this highly selected group of colonoscopists in the 12 months after they commenced screening. 139,363 procedures were performed between June 2006 and March 2012 by 245 colonoscopists. Data were collected on caecal intubation rate (CIR), the adenoma detection rate $(\mathrm{ADR})$, the mean number of adenomas detected per patient (MAP) and the number of procedures performed by each colonoscopist.

Results Colonoscopists were divided into quartiles by performance for CIR and ADR. The mean CIR in the top quartile was $98.6 \%$ compared to $91.4 \%$ in the lowest quartile. The mean number of procedures in the first year of screening was 141 and 123 respectively ( $t$-test $p=0.055)$; there was a correlation between the number of procedures performed and CIR (Pearson's $r=0.196, p=0.002$ ). There was a significant difference in the number of procedures performed in the previous year before commencing screening (266 compared to 201, $\mathrm{p}=0.02$ ). There was no significant difference in the $\operatorname{ADR}(p=0.48)$, pain caused, years of experience or number of lifetime procedures. There was no correlation between CIR and $\operatorname{ADR}(r=0.001, p=0.982)$. The mean ADR in the top quartile was $56.8 \%$ compared to $39.8 \%$ in the lowest quartile. There was no significant difference between these groups in the CIR, pain or any other measure from the lifetime procedure log. There was a significant difference in the MAP (1.64 compared to 0.77, $\mathrm{p}<0.01)$. Overall there was a strong correlation between ADR and MAP ( $r=0.59$, $\mathrm{p}<0.001)$

Conclusion These data show that even in a stratified group of high-performing colonoscopists there is considerable variation in performance in CIR, ADR and MAP. There was no correlation between CIR and ADR but a significant association between CIR and the endoscopic activity the previous year. Colonoscopists performing more procedures in their first year screening patients did not have higher adenoma detection rates. Further studies are needed to ascertain the factors responsible for these differences to try to further improve performance and patient outcomes.

Disclosure of Interest None Declared.

\section{PWE-049 FIVE YEAR RETROSPECTIVE MORBIDITY AND MORTALITY FIGURES FOR COLONOSCOPY IN A DISTRICT GENERAL HOSPITAL}

doi:10.1136/gutjnl-2013-304907.338

\section{1."K M Barnett, 'C Gordon, 'S Weaver. 'Gastroenterology, Royal Bournemouth Hospital, Bournemouth, UK}

Introduction Between 1 July 2007 and 31 August 2012, 15284 colonoscopies were performed by Gastroenterologists, Colorectal Surgeons, Gastroenterology and Surgical trainees and Nurse Endoscopists. Over the 5 year period our unit has an average caecal intubation rate of $93.53 \%$ and polyp detection rate of $36.54 \%$ demonstrating colonoscopy performance that meets current national guidelines for performance indicators in endoscopy. The current JAG guidelines for complications of colonoscopy are perforation rate $<1: 1000$, post polypectomy bleeding requiring transfusion $<1: 100$ and post polypectomy perforation $<1: 500$.

Aim To determine the complication rate and 30 day mortality of colonoscopy performed in a district general hospital (DGH).

Methods Using the hospital IT system and a coding search, patients were identified if they had died within 30 days, were readmitted or went to theatre within 8 days of a colonoscopy. Case notes were then reviewed to assess if any event was directly related to the procedure or intervention undertaken.

Results In the 62 month period one death occurred from sepsis due to perforated diverticular disease (DD) (1/15284). In this time frame 4023 patients were diagnosed with DD giving a risk for perforation and death in DD of 1:4000.

Perforation: occurred in 13 patients (1:1175), the causes listed in table 1.

Abstract PWE-049 Table 1 Causes of perforation

\begin{tabular}{lll}
\hline Cause & Number & Rate for finding/intervention \\
\hline DD & 4 & $4 / 4023$ DD - 1:1000 \\
Polypectomy & 6 & 6/5966 pts - 1:1000 \\
APC & 2 & $2 / 115$ APC - 1:63 \\
Retroflexion post APR & 1 & \\
\hline
\end{tabular}

One patient with Crohn's disease of the terminal ileum (TI) developed severe pain post procedure requring a laparotomy where the cause was found to be tearing of an adhesion to the TI, no perforation was seen $1 / 15284$

Bleeding Polyps were found in 5966 patients. Post polypectomy bleeding requiring admission occurred in 27 patients (1:220 patients). 18 were observed for 24-48 hours without any intervention. 9 required transfusion (1:663). Of these patients, one required a right hemicolectomy for haemostasis and one required angiogram and embolisation, the remaining managed conservatively. Post biopsy bleeding occurred in 4 patients out of 8702 who were biopsied (1:2175)

Post polypectomy syndrome occurred in 3 patients of 5966 patients with polyps (1:2000)

Pain post colonoscopy requiring admission occurred in 12 patients (1:1273). Pain was attributed to air insufflation with radiology excluding perforation.

Bowel preparation with Fleet resulted in one patient developing end stage renal failure due to phosphate nephropathy (1:4715), This preparation is no longer in use.

Conclusion Few papers are published on the complication rates of colonoscopy at DGHs. This data gives valuable long term insight into the rate of serious complications and demonstrates safe colonoscopy at this DGH within current JAG Guidelines.

Disclosure of Interest None Declared.

\section{PWE-050 AN EVALUATION OF PATIENTS REFERRED TO GASTROENTEROLOGY ANAEMIA CLINIC}

doi:10.1136/gutjinl-2013-304907.339

1.*L Chinnappan, 'S Westall, 'P Hayle, 'I London. 'Gastroenterology, Leighton Hospital NHS Trust, Crewe, UK

Introduction Iron-deficiency anaemia in adults is a common cause of referral to gastroenterologists (4-13\% of referrals). NICE guidelines, UK provides clear recommendations regarding upper and lower gastrointestinal investigations for such patients including coeliac and urine screening. The aim of this audit is to conduct a snapshot review of anaemic patients seen in gastroenterology anaemia clinic conducted by specialist nurses with consultant gastroenterologists input in a DGH to assess adherence to the NICE guidelines and to highlight changes in clinical practise that may be required, with implications for service development.

Methods Retrospective study involving 90 new patients referred predominantly from primary health care $(92 \%)$ between July to September 2012.Of the 90 patients referred, 84 patients in the age range of 30-90 attended the clinic of which 53\% were females.

Results The mean $\mathrm{HB}$ was 10.8 \& $80 \%$ of the patients had ferritin level of less than 45 . Of them 14 patients (16\%), all referred from 


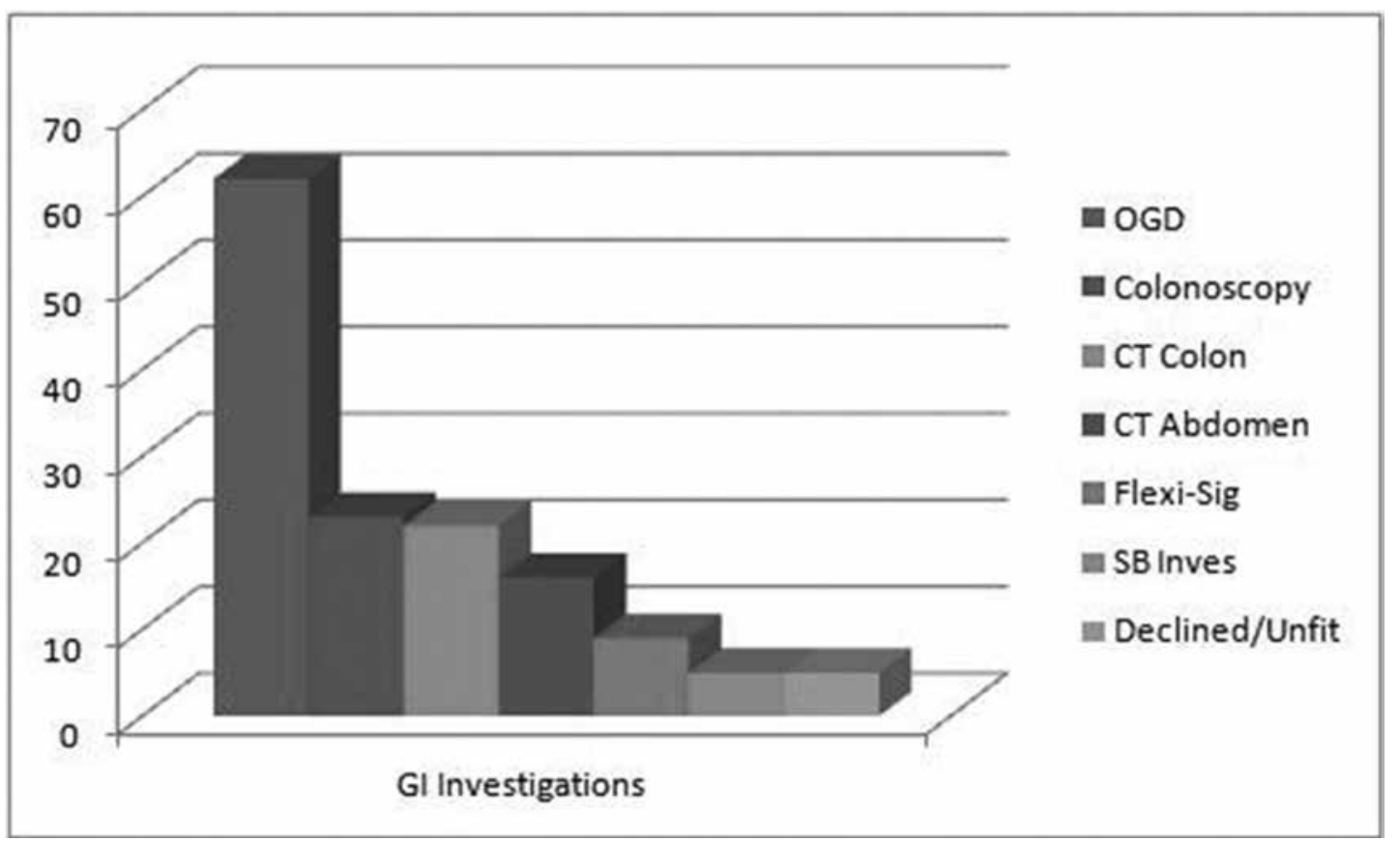

\section{Abstract PWE-050 Figure}

primary care were normocytic with no evidence of iron deficiency and did not require GI investigations. All iron deficient patients underwent coeliac screening (100\%) but only $62 \%$ had evidence of urine screening for blood loss. $82 \%$ of the patients finished their investigations within 12 weeks from their first clinic appointment.62 patients (88\%) underwent upper GI endoscopy and the rest were either unfit or declined endoscopies. Lower gastrointestinal tract was investigated in $75 \%$ of patients endoscopically or by CT colon (25\%) and 22\% underwent CT abdomen. An upper GI cause for anaemia was found in 30 patients (42\%) and $20 \%$ had lower GI cause with gastric and colonic cancer diagnosed in one patient each. 5 patients (22\%) with resistant anaemia are awaiting small bowel investigations whilst the rest $(21 \%)$ are being monitored on iron therapy. Interestingly one patient each with lung, renal and bone malignancy were diagnosed and one with carcinoid after undergoing investigations in this clinic.

\section{Conclusion}

1. Development of a referral pro-forma to anaemia clinic with clear criteria to establish iron deficiency has been recommended to ensure appropriate referrals and to enable effective use of resources. The importance of regular urine screening and its inclusion in the referral pro-forma to assess renal causes for anaemia is being highlighted.

2. Though the detection rate of gastrointestinal malignancy in anaemia clinic during the audit period was low, this rate can be periodically variable. The audit found that majority of patients with iron deficiency anaemia underwent upper and lower GI investigations in compliance with NICE guidelines within a satisfactory time scale and investigations not only led to early detection of GI cancers but also other malignancies.

Disclosure of Interest None Declared.

\section{REFERENCE}

NICE Guidelines UK for Iron deficiency anaemia.

\section{PWE-051 WHAT IS THE TRUE INCIDENCE OF LYMPHOCYTIC AND COLLAGENOUS COLITIS?}

doi:10.1136/gutjnl-2013-304907.340

1."L Stevens, 'G Kaur. 'Surgery, Scunthorpe General Hospital, Scunthorpe, UK
Introduction Increased incidence of both lymphocytic (LC) and collagenous (CC) colitis, often described under the umbrella term of microscopic colitis (MC), is being seen globally. The aetiology, pathogenesis and incidence of $\mathrm{MC}$ is yet to be well established. However, not all patients with diarrhoea undergo biopsies to cheque for MC. We aimed to investigate our patient population attending for colonoscopy with symptoms of diarrhoea to assess the proportion of patients undergoing random colon/rectal biopsies to look for $\mathrm{MC}$ as well as the incidence of LC/CC in our population.

Methods Retrospective analysis of the endoscopy database was performed to identify patients presenting from January - December 2011 for colonoscopy with diarrhoea. Patients with a complete colonoscopy with macroscopically normal mucosa or findings unrelated to symptoms were included. MC patients were identified by histology.

Results A total of 939 colonoscopies were performed during this period for either diarrhoea or undocumented symptoms. Of these, 186 were for patients with definite diarrhoea and were both complete as well as macroscopically normal. Of these, $123(66 \%)$ had random biopsies taken. 2 patients i.e 1\% of all diarrhoea patients undergoing colonoscopy (1.6\% of all diarrhoea patients undergoing colonoscopy and random biopsies) were diagnosed with CC while 5 patients i.e $2.7 \%$ of all diarrhoea patients undergoing colonoscopy (4.1\% of all diarrhoea patients undergoing colonoscopy and random biopsies) were diagnosed with LC. Thus, $3.8 \%$ of all diarrhoea patients undergoing colonoscopy were diagnosed with MC $(5.7 \%$ of all diarrhoea patients undergoing colonoscopy and random biopsies). Conclusion Though there appears to be an increase in incidence of $\mathrm{MC}$ documented in literature, we did not find this in our endoscopy unit. This may be due to variability in endoscopists resulting in inadequate random biopsies being undertaken during colonoscopy performed to investigate diarrhoea. We are currently undertaking an audit to ensure improved management of these patients as well as improve our accuracy in the assessment of the true incidence of $\mathrm{MC}$ Disclosure of Interest None Declared.

\section{REFERENCE}

1. Fernández-Bañares F, Salas A, Forné M, Esteve M, Espinós J, Viver JM. Incidence of collagenous and lymphocytic colitis: a 5-year population-based study. Am J Gastroenterol. 1999 Feb; 94(2):418-23. 